Bangladesh J. Pl. Breed. Genet., 27(2): 01-08, 2014

\title{
CHARACTERIZATION OF BRASSICA SPP. PROGENY LINES FOR YIELD AND ITS RELATED TRAITS
}

\author{
A. Iqbal, L. Rahman ${ }^{1}$ and M. H. Kabir \\ Department of Biotechnology \\ Bangladesh Agricultural University, Mymensingh 2202, Bangladesh \\ ${ }^{1}$ Department of Plant Breeding and Genetics, Bangladesh Agricultural University, Mymensingh \\ 2202, Bangladesh
}

\begin{abstract}
The present research works were conducted to find out the variation in morphological traits and their relationship in Brassica materials at $\mathrm{C}_{7}$ (Colchiploid) alloplasmic populations and $\mathrm{F}_{5}$ generations with respect to their parents. Among the 19 Brassica progeny lines representing the cross products (Brassica napojuncea) of $\mathrm{C}_{7}$ generation of Brassica napus and Brassica juncea (BINA sharisha $5 \times$ Daulot: colchiploid- $\mathrm{C}_{7}$ ) and $\mathrm{F}_{5}$ generations with their parents, inter-species $\mathrm{EG}_{2}$ (Brassica napus $\mathrm{x}$ Brassica juncea) and $\mathrm{EG}_{1}$ (Brassica napus x Brassica juncea) showed better agronomic performance. Phenotypic characters of the genotypes were closely associated with each other for both normal correlation coefficients and Spearman's rank correlation coefficients. The total relative ranking ranged from 1 to 20 and the first rank was estimated for the genotype $\mathrm{EG}_{2}$ genotypes. The last rank was estimated for the genotype $\mathrm{ED}_{1}$ (Brassica napus x Brassica napus). Out of 55 associations, which found in the correlation study, 10 associations were found highly significant at the morphological level. Flower set per plant (self) showed highly significant positive association with total number of siliqua per plant (self) $\left(\mathrm{r}=0.934^{* *}\right)$ but the significant negative association of the total number of siliqua per plant (self) with siliqua length was very strong $\left(-0.489^{*}\right)$. There was a genetic basis of association in most of the cases of pairs of relations. The Spearman's rank correlation test was studied to compare the morphological characterization for distinguishing genotypes. In this present study, this test presented 12 relationships that showed 10 positive significant and two negative significant correlations. In normal correlation coefficients 10 significant associations were found but 12 associations in Spearman's rank correlation. In these techniques, number of flower set per plant (self) had highly significant positive correlation with total number of siliqua per plant $\left(\mathrm{r}_{\mathrm{s}}=0.817^{* *}\right)$. The days to $50 \%$ flowering and number of seed per siliqua (self) had significant negative correlation with number of seed per siliqua (open) $\left(r_{s}=-0.546^{* *}\right)$ and number of flowers set per plant (open) $\left(r_{s}=-0.563^{*}\right)$, respectively. Finally, the study suggested that some yield related traits such as flower set per plant, number of siliqua per plant, seed per siliqua and days to $50 \%$ flowering can be selected and used in breeding program for future increasing the grain yield of rapeseed.
\end{abstract}

Key words: Brassica; interspecific hybridization; morphological traits; characterization

DOI: http://dx.doi.org/10.3329/bjpbg.v27i2.27843

\section{INTRODUCTION}

Rapeseed and mustard are the top ranking oilseed crops in Bangladesh and it is a significant source of cooking oil that meets up one-third of the total edible oil 
requirements in the country (Ahmed, 2008). In addition, it is a high-energy source along with a suitable content of fat-soluble vitamins (A, D, E and K) for our human body. In Bangladesh, the total cultivated land covered since 2011-12 was 0.483 million hectares with production and yield of 0.525 million Mt and 0.109 million $\mathrm{Mt} \mathrm{ha}^{-1}$ respectively (AIS, 2013). Ultimately, it covers the highest $68 \%$ of the total oil seed acreage of Bangladesh (BBS, 2011). Moreover, this production covered only $40 \%$ of the total oil requirements of Bangladesh as per recommended dietary allowances (RDA). But unfortunately, Bangladesh has been fronting extreme deficit of edible oil during the last several decades due to fact that our domestic production cannot meet-up the total dietary requirements but only about $21 \%$ of the total consumption and rest of the portion (79\%) filled-up by imports from abroad (Begum et al. 2012). Moreover to meet-up the high demand of the edible oil in Bangladesh, it imports 0.08997 million tons of edible oil that costs massive amount of money (371.85 million BDT) (BBS, 2011). And in Bangladesh, there is very limited scope to increase acreage due to heavy pressure of other crops, cropping patterns and human population also. Therefore, sustainable attempts should be taken to develop high yielding variety (HYV) and upsurge their adaptation in different areas of Bangladesh for cultivation. In Bangladesh, more than 18 varieties of rapeseed and mustard were developed by different organizations. And the rapeseed and mustard varieties were developed through introduction, hybridization and mutation breeding programs at both inter-varietal and inter-specific level (Naz et al., 2003). But in order to improve the hybridization of varieties both at inter-variety and inter-species level it could be effective when appropriate selection techniques will be followed. In general, the main objective of my current research is to develop varieties with higher seed yield with sufficient oil content and quality. And then breeding for improvement of protein content, resistance against diseases, insects, shattering and lodging have more or less been neglected. So, the present study was conducted to characterize the morphological traits of $\mathrm{C}_{7}$ and $\mathrm{F}_{5}$ generation of Brassica inter-species and intra-species progeny lines along with their parents.

\section{MATERIALS AND METHODS}

The experiment was conducted at the field laboratory of the Department of Genetics and Plant Breeding, Bangladesh Agricultural University, Mymensingh, during the winter season. To study the morphological characteristic of 19 Brassica progeny lines representing the cross products (Brassica napojuncea) of the $\mathrm{C}_{7}$ generation of Brassica napus and Brassica juncea (BINA sharisha $5 \mathrm{x}$ Daulot : colchiploid- $\mathrm{C}_{7}$ ) and the intra-species $\mathrm{F}_{5}$ generation between two different varieties of $B$. napus (BINA sharisha 4 x BINA sharisha 5 and its reciprocal); and parents were used. The field was first ploughed in November and all stubbles were removed from the experimental field. Four ploughing and cross ploughing followed by laddering were given during land preparation to ensure good tilth. During the initial land preparation, only cowdung and at the final land preparation Urea (first of splits $)(132 \mathrm{~g}+13.2 \mathrm{~g})$, Triple Super Phosphate (TSP 15g) and Muroate of Potash (MP 9.6g), Zinc oxide(3.24g), Gypsum(13.44g) and Borax $(1.63 \mathrm{~g})$ per plot of $1.2 \mathrm{~m}^{2}$ were applied. The seeds of four interspecies $\left(\mathrm{C}_{6}\right.$ geneation lines) and 15 intraspecies $\left(\mathrm{F}_{4}\right.$ generation lines) progeny lines were sown in separate rows along with their parents to facilatate selection of appropriate types at a depth of $2.5 \mathrm{~cm}$. The row size was $3.6 \mathrm{~m}$. The distance between rows were $40 \mathrm{~cm}$ and between plants $5 \mathrm{~cm}$ for proper development and growth of plants. Intercultural operations such as thinning, weeding application of irrigation insecticides, etc. were done uniformly when necessary in order to maintain proper growth conditions. Data were taken from nine randomly selected plants of each progeny lines and three plants for 
selfing in the same progeny lines. The selection was done on the basis of plants having bigger leaves, good pod formation, plant structure, canopy etc. This research was aiming to select plants with higher ploidy position and estimates of morphometric traits of those to compare with that of their parents. Data were recorded on days to $50 \%$ flowering, number of flowers set per plant (open), number of flowers set per plant (self), number of siliqua per plant (open), number of siliqua per plant (self), number of seeds per siliqua (open), number of seeds per siliqua (self), siliqua length $(\mathrm{cm})$, beak length $(\mathrm{cm})$, plant height at maturity, 1000-seed weight (g) from the sample plants. The recorded data were statistically analyzed using MSTAT-C, a computer based statistical package.

\section{RESULTS AND DISCUSSION}

In this study, there were phenotypic variations between Daulot and other genotypes. Daulot produced smaller beak length; less number of seeds per siliqua and 1000 seeds weight were also less than other genotypes. At the cross combination of the two species, it was found that the maximum number of days to $50 \%$ flowering was required for inter-species EG (Brassica napus x Brassica juncea) which is $4.79 \%$ higher than their parents. That indicates $B$. juncea which have had more control over this character when in cross combination with $B$. napus. In case of self pollination, interspecies EG ( $B$. napus x $B$. juncea) progeny lines showed the better performance than their respective parents and other crosses in number of flower set per plant. $B$. napojuncea produced more siliqua in the other crosses and also produced more siliqua than the B. napus and B. juncea parents. In case of open pollination, crossed materials performed better than their parents. Number of flower set per plant and total number of siliqua per plant in EG (Brassica napus x Brassica juncea) and DE (Brassica napus $\mathrm{x}$ Brassica napus) progeny lines showed higher percentage over their parents. But the lowest total number of siliqua per plant was found in the cross ED (Brassica napus $\mathrm{x}$ Brassica napus) (2.66 siliqua per plant) and it showed $27.32 \%$ lower performance than their parents. Number of seeds per siliqua in open pollination was found and was comparatively $42.82 \%$ higher than the self-pollination. In case of open pollination, number of seeds per siliqua of crossed materials was much higher than their parents. This result proved that in the open pollination, the species are influenced by other species and for that they showed the higher number of seeds than the self-pollinated species. In case of siliqua, length of EG progeny lines showed the better performance than their respective parents and that was $19.93 \%$ higher than their parents. Inter-species EG progeny lines also showed better performance in beak length, plant height and 1000-seed weight and they showed $17.77 \%, 10.16 \%$ and $18.37 \%$ higher than their parents, respectively (Table 1$)$.

The results of correlation coefficients among 1000-seed weight and yield contributing characters in all possible combinations were made for the inter and intra species genotypes and their parents are presented in Table 2. Out of 55 associations 10 associations were highly significant at the morphological level. The strong and positive associations between the characters suggested additive genetic model thereby less affected by environmental fluctuation. On the other hand 18 relationships were found negative and non-significant. The positive and non-significant association referred information of inherent relation among the pairs of combination. While the negative and non-significant association referred a complex linked of relation among pair of combinations. Number of flower set per plant (self) had highly significant positive correlation with total number of siliqua per plant (self) $\left(\mathrm{r}=0.934^{* *}\right)$ and plant height $(\mathrm{r}$ $\left.=0.546^{* *}\right)$. 
Table 1. Performances of yield and yield components of intra and inter species Brassica progeny lines (EG (Brassica napus $\mathrm{x}$ Brassica juncea); DE (Brassica napus $\mathrm{x}$ Brassica napus) ; ED (Brassica napus $\mathrm{x}$ Brassica napus))

\begin{tabular}{|c|c|c|c|c|c|c|c|c|c|c|c|c|}
\hline \multirow{2}{*}{$\begin{array}{l}\text { Serial } \\
\text { No. }\end{array}$} & \multirow{2}{*}{$\begin{array}{l}\text { Progeny } \\
\text { line }\end{array}$} & \multirow{2}{*}{$\begin{array}{l}50 \% \\
\text { flowering } \\
\text { days }\end{array}$} & \multicolumn{3}{|c|}{ Self pollination } & \multicolumn{7}{|c|}{ Open pollination } \\
\hline & & & $\begin{array}{l}\text { No. flower } \\
\text { set per } \\
\text { plant / day }\end{array}$ & $\begin{array}{l}\text { Total no. } \\
\text { siliqua per } \\
\text { plant }\end{array}$ & $\begin{array}{l}\text { No. seeds per } \\
\text { siliqua }\end{array}$ & $\begin{array}{l}\text { No. flower set } \\
\text { per plant/day }\end{array}$ & $\begin{array}{l}\text { Total no. } \\
\text { siliqua per } \\
\text { plant }\end{array}$ & $\begin{array}{l}\text { No. seeds per } \\
\text { siliqua }\end{array}$ & $\begin{array}{l}\text { Siliqua length } \\
(\mathrm{cm})\end{array}$ & $\begin{array}{l}\text { Beak length } \\
(\mathrm{cm})\end{array}$ & $\begin{array}{l}\text { Plant height } \\
(\mathrm{cm})\end{array}$ & $\begin{array}{l}1000-\text { seed } \\
\text { weight }(\mathrm{gm})\end{array}$ \\
\hline \multicolumn{13}{|c|}{ Inter species lines } \\
\hline 1 & $\mathrm{EG}_{1}$ & 48 & 9.66 & 8.33 & 18.00 & 14.11 & 78.77 & 16.44 & 4.44 & 1.10 & 95.44 & 4.15 \\
\hline 2 & $\mathrm{EG}_{2}$ & 47 & 10.66 & 8.66 & 18.00 & 11.00 & 88.77 & 29.22 & 4.31 & 1.03 & 95.66 & 3.76 \\
\hline 3 & $\mathrm{EG}_{3}$ & 50 & 5.00 & 5.00 & 20.66 & 8.33 & 71.55 & 24.31 & 5.69 & 1.05 & 89.44 & 3.72 \\
\hline 4 & $\mathrm{EG}_{4}$ & 52 & 7.33 & 6.33 & 22.66 & 11.44 & 66.88 & 19.00 & 4.95 & 1.05 & 102.00 & 4.10 \\
\hline \multicolumn{2}{|l|}{ Mean } & $49.25(4.79 \%)$ & $8.16(81.33 \%)$ & $7.08(70.19 \%)$ & $19.83(18.50 \%)$ & $11.22(2.73 \%)$ & $76.49(6.71 \%)$ & $22.24(5.55 \%)$ & $4.85(19.93 \%)$ & $1.06(17.77 \%)$ & $95.64(10.16 \%)$ & $3.93(18.37 \%)$ \\
\hline \multicolumn{2}{|l|}{ SD } & 2.22 & 2.53 & 1.73 & 2.27 & 2.37 & 9.54 & 5.69 & 0.63 & 0.03 & 5.13 & 0.22 \\
\hline \multirow{2}{*}{\multicolumn{13}{|c|}{$\begin{array}{l}\text { CV\% } \\
\text { Intra species lines }\end{array}$}} \\
\hline & & & & & & & & & & & & \\
\hline 5 & $\mathrm{DE}_{1}$ & 50 & 4.66 & 4.66 & 20.66 & 12.66 & 86.44 & 23.88 & 5.24 & 1.00 & 93.22 & 4.04 \\
\hline 6 & $\mathrm{DE}_{2}$ & 47 & 4.00 & 3.00 & 12.66 & 13.22 & 96.55 & 29.60 & 6.16 & 1.04 & 87.44 & 3.75 \\
\hline 7 & $\mathrm{DE}_{3}$ & 45 & 4.00 & 2.66 & 8.66 & 13.44 & 65.88 & 21.11 & 5.10 & 0.86 & 80.88 & 3.97 \\
\hline 8 & $\mathrm{DE}_{4}$ & 50 & 5.00 & 4.33 & 20.66 & 9.00 & 74.77 & 21.75 & 5.77 & 1.13 & 97.77 & 3.77 \\
\hline 9 & $\mathrm{DE}_{5}$ & 45 & 4.33 & 2.00 & 12.66 & 9.88 & 75.55 & 25.17 & 5.79 & 1.26 & 91.11 & 3.00 \\
\hline 10 & $\mathrm{DE}_{6}$ & 46 & 4.00 & 3.33 & 13.33 & 11.55 & 86.22 & 25.35 & 5.81 & 1.19 & 96.77 & 3.19 \\
\hline 11 & $\mathrm{DE}_{7}$ & 45 & 3.33 & 3.00 & 13.33 & 21.33 & 81.44 & 23.04 & 6.01 & 1.14 & 85.00 & 3.91 \\
\hline 12 & $\mathrm{DE}_{8}$ & 47 & 4.00 & 3.33 & 12.00 & 19.33 & 69.44 & 25.54 & 5.85 & 0.94 & 86.77 & 3.26 \\
\hline 13 & $\mathrm{DE}_{9}$ & 48 & 5.00 & 3.66 & 12.66 & 19.00 & 54.66 & 23.42 & 5.64 & 0.96 & 84.22 & 4.27 \\
\hline 14 & $\mathrm{DE}_{10}$ & 59 & 4.66 & 4.00 & 14.66 & 9.11 & 60.66 & 21.13 & 5.44 & 0.94 & 89.33 & 3.25 \\
\hline \multicolumn{2}{|l|}{ Mean } & $48.20(7.11 \%)$ & $4.30(0.69 \%)$ & $3.40(7.10 \%)$ & $14.13(50.70 \%)$ & $13.85(41.62 \%)$ & $75.16(6.28 \%)$ & $23.99(3.85 \%)$ & $5.68(18.89 \%)$ & $1.05(0.96 \%)$ & $89.25(18.84 \%)$ & $3.64(2.67 \%)$ \\
\hline \multicolumn{2}{|l|}{ SD } & 4.24 & 0.53 & 0.80 & 3.77 & 4.49 & 12.89 & 2.58 & 0.33 & 0.13 & 5.49 & 0.43 \\
\hline CV\% & & 8.791 & 12.363 & 23.463 & 26.671 & 32.422 & 17.154 & 10.745 & 5.851 & 12.303 & 6.151 & 11.853 \\
\hline \multicolumn{13}{|c|}{ Intra reciprocal species lines } \\
\hline 15 & $\mathrm{ED}_{1}$ & 48 & 3.00 & 2.00 & 10.00 & 13.44 & 65.88 & 16.00 & 5.03 & 0.95 & 84.00 & 3.28 \\
\hline 16 & $\mathrm{ED}_{2}$ & 50 & 3.66 & 1.33 & 6.00 & 15.11 & 56.33 & 25.24 & 5.30 & 0.78 & 72.55 & 4.06 \\
\hline 17 & $\mathrm{ED}_{3}$ & 48 & 3.00 & 2.66 & 8.00 & 12.44 & 62.55 & 24.35 & 5.12 & 0.90 & 76.33 & 3.15 \\
\hline 18 & $\mathrm{ED}_{4}$ & 50 & 3.66 & 3.33 & 10.00 & 13.44 & 44.88 & 20.48 & 5.36 & 0.96 & 73.44 & 3.47 \\
\hline 19 & $\mathrm{ED}_{5}$ & 50 & 4.00 & 4.00 & 14.00 & 12.00 & 46.44 & 21.97 & 5.08 & 0.96 & 70.66 & 3.51 \\
\hline \multicolumn{2}{|l|}{ Mean } & $49.20(9.33 \%)$ & $3.46(20.09 \%)$ & $2.66(27.32 \%)$ & $9.60(66.50 \%)$ & $13.29(35.80 \%)$ & $55.22(21.92 \%)$ & $21.61(6.45 \%)$ & $5.18(2.98 \%)$ & $0.91(12.5 \%)$ & $75.40(0.40 \%)$ & $3.49(6.68 \%)$ \\
\hline \multicolumn{2}{|l|}{ SD } & 1.10 & 0.45 & 1.05 & 2.97 & 1.20 & 9.39 & 3.66 & 0.14 & 0.08 & 5.23 & 0.35 \\
\hline CV\% & & 2.227 & 12.868 & 39.588 & 30.901 & 9.019 & 17.004 & 16.935 & 2.780 & 8.441 & 6.932 & 9.969 \\
\hline \multicolumn{13}{|c|}{ Parents line } \\
\hline 20 & $\mathrm{E}$ & 42 & 4.00 & 3.66 & 25.33 & 18.11 & 66.33 & 26.40 & 5.17 & 0.98 & 78.88 & 3.81 \\
\hline 21 & $\mathrm{D}$ & 48 & 4.66 & 3.66 & 32.00 & 20.44 & 75.11 & 19.80 & 4.88 & 1.10 & 71.33 & 3.66 \\
\hline$\frac{21}{22}$ & $\mathrm{G}$ & 52 & $\begin{array}{l}5.00 \\
5.00\end{array}$ & $\begin{array}{l}4.00 \\
.66\end{array}$ & 23.33 & $\begin{array}{l}23.44 \\
23.04\end{array}$ & $\begin{array}{l}1.11 \\
77.02\end{array}$ & 15.00 & $\begin{array}{l}4.00 \\
3.27\end{array}$ & 0.82 & 94.76 & 2.00 \\
\hline \multirow{2}{*}{\multicolumn{2}{|c|}{ Total Mean }} & 48.50 & 4.85 & 3.98 & 15.88 & 12.81 & 70.55 & 22.68 & 5.25 & 1.01 & 86.23 & 3.63 \\
\hline & & 3.39 & 1.95 & 1.83 & 6.45 & 3.41 & 13.33 & 3.81 & 0.65 & 0.19 & 9.36 & 0.41 \\
\hline Total CV \% & Total SD & 6.992 & 40.235 & 45.948 & 40.621 & 26.657 & 18.894 & 16.797 & 12.373 & 11.719 & 10.856 & 11.150 \\
\hline
\end{tabular}


Table 2. Correlation coefficients among yield related characters of 22 genotypes of Brassica Spp.

\begin{tabular}{|c|c|c|c|c|c|c|c|c|c|c|}
\hline Characters & $\begin{array}{c}\text { No. of } \\
\text { flower set } \\
\text { per plant } \\
\text { (self) }\end{array}$ & $\begin{array}{l}\text { Total no. } \\
\text { siliqua per } \\
\text { plant } \\
\text { (self) }\end{array}$ & $\begin{array}{c}\text { No. of } \\
\text { seeds per } \\
\text { Siliqua } \\
\text { (self) }\end{array}$ & $\begin{array}{c}\text { No. of } \\
\text { flower set } \\
\text { per plant } \\
\text { (open) }\end{array}$ & $\begin{array}{l}\text { Total no. } \\
\text { siliqua per } \\
\text { plant } \\
\text { (open) }\end{array}$ & $\begin{array}{c}\text { No. of } \\
\text { seeds per } \\
\text { Siliqua } \\
\text { (open) }\end{array}$ & $\begin{array}{l}\text { Siliqua } \\
\text { length } \\
(\mathrm{cm})\end{array}$ & $\begin{array}{l}\text { Beak } \\
\text { length } \\
(\mathrm{cm})\end{array}$ & $\begin{array}{l}\text { Plant } \\
\text { height } \\
(\mathrm{cm})\end{array}$ & $\begin{array}{c}1000 \text { seed } \\
\text { weight } \\
\text { (gm) }\end{array}$ \\
\hline $50 \%$ flowering (days) & 0.098 & 0.176 & 0.064 & -0.252 & -0.280 & -0.384 & -0.211 & -0.302 & 0.162 & -0.139 \\
\hline No. of flower set per plant (self) & & $0.934 * *$ & 0.361 & -0.172 & 0.328 & -0.040 & $-0.473 *$ & 0.213 & $0.546 * *$ & 0.349 \\
\hline Total no. siliqua per plant (self) & & & $0.486 *$ & -0.205 & 0.311 & -0.111 & $-0.489 *$ & 0.199 & $0.553 * *$ & 0.308 \\
\hline No. of seeds per Siliqua (self) & & & & $-0.454 *$ & 0.318 & -0.197 & -0.363 & 0.298 & 0.261 & 0.110 \\
\hline No. of flower set per plant (open) & & & & & -0.076 & 0.009 & 0.203 & -0.194 & -0.164 & 0.253 \\
\hline Total no. siliqua per plant (open) & & & & & & 0.305 & 0.020 & $0.488 *$ & $0.608 * *$ & 0.011 \\
\hline No. of seeds per siliqua (open) & & & & & & & $0.527 *$ & 0.164 & -0.054 & 0.100 \\
\hline Siliqua length $(\mathrm{cm})$ & & & & & & & & 0.404 & -0.098 & 0.142 \\
\hline Beak length $(\mathrm{cm})$ & & & & & & & & & 0.422 & -0.004 \\
\hline Plant height $(\mathrm{cm})$ & & & & & & & & & & 0.010 \\
\hline
\end{tabular}

$*$ and $* *$ indicate significant at $5 \%$ and $1 \%$ level of probability, respectively 
Table 3. Relative ranking of genotypes on the basis of yield related trait

\begin{tabular}{|c|c|c|c|c|c|c|c|c|c|c|c|c|c|c|c|c|c|c|c|c|c|c|c|c|}
\hline \multirow{2}{*}{\multicolumn{2}{|c|}{ Characters }} & \multirow{2}{*}{\multicolumn{2}{|c|}{$\begin{array}{l}50 \% \\
\text { floweri } \\
\text { ng } \\
\text { (days) }\end{array}$}} & \multicolumn{6}{|c|}{ Self pollination } & \multicolumn{14}{|c|}{ Open pollination } & \multirow{3}{*}{ 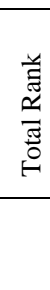 } \\
\hline & & & & \multicolumn{2}{|c|}{$\begin{array}{l}\text { No. flower } \\
\text { set per } \\
\text { plant }\end{array}$} & \multicolumn{2}{|c|}{$\begin{array}{l}\text { Total no. } \\
\text { of siliqua } \\
\text { per plant }\end{array}$} & \multicolumn{2}{|c|}{$\begin{array}{c}\text { No. of } \\
\text { seeds } \\
\text { per siliqua }\end{array}$} & \multicolumn{2}{|c|}{$\begin{array}{c}\text { No. flower } \\
\text { set per } \\
\text { plant }\end{array}$} & \multicolumn{2}{|c|}{$\begin{array}{l}\text { Total no. } \\
\text { of siliqua } \\
\text { per plant }\end{array}$} & \multicolumn{2}{|c|}{$\begin{array}{c}\text { No. of } \\
\text { seeds } \\
\text { per siliqua }\end{array}$} & \multicolumn{2}{|c|}{$\begin{array}{l}\text { Siliqua } \\
\text { length } \\
(\mathrm{cm})\end{array}$} & \multicolumn{2}{|c|}{$\begin{array}{c}\text { Beak } \\
\text { length } \\
(\mathrm{cm})\end{array}$} & \multicolumn{2}{|c|}{$\begin{array}{c}\text { Plant } \\
\text { Height } \\
(\mathrm{cm})\end{array}$} & \multicolumn{2}{|c|}{$\begin{array}{l}\text { 1000-seed } \\
\text { weight } \\
(\mathrm{gm})\end{array}$} & \\
\hline 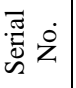 & 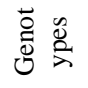 & 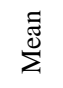 & 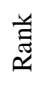 & $\sum_{\Sigma}^{\bar{E}}$ & 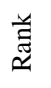 & 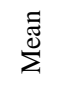 & 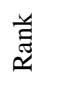 & 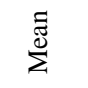 & 弟 & 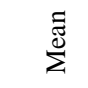 & $\begin{array}{l}\text { 婷 } \\
\text { œ }\end{array}$ & $\stackrel{\mathrm{\Xi}}{\Sigma}_{\Sigma}$ & 弟 & 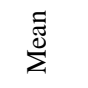 & $\begin{array}{l}\text { 弟 } \\
\text { 出 }\end{array}$ & $\stackrel{\bar{E}}{\Sigma}$ & $\begin{array}{l}\text { 蓔 } \\
\simeq\end{array}$ & 丞 & $\begin{array}{l}\text { 弟 } \\
\text { 品 }\end{array}$ & $\stackrel{\Xi ّ}{\Sigma}$ & Еี & 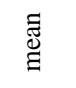 & & \\
\hline 1 & $\mathrm{EG}_{1}$ & 48 & 5 & 9.66 & 2 & 8.33 & 2 & 18.00 & 6 & 14.11 & 13 & 78.77 & 6 & 16.44 & 20 & 4.44 & 20 & 1.10 & 5 & 95.44 & 5 & 4.15 & 2 & 9 \\
\hline 2 & $\mathrm{EG}_{2}$ & 47 & 4 & 10.66 & 1 & 8.66 & 1 & 18.00 & 6 & 11.00 & 5 & 88.77 & 2 & 29.22 & 2 & 4.31 & 21 & 1.03 & 8 & 95.66 & 4 & 3.76 & 10 & 1 \\
\hline 3 & $\mathrm{EG}_{3}$ & 50 & 6 & 5.00 & 4 & 5.00 & 4 & 20.66 & 5 & 8.33 & 1 & 71.55 & 11 & 24.31 & 9 & 5.69 & 7 & 1.05 & 6 & 89.44 & 9 & 3.72 & 12 & 4 \\
\hline 4 & $\mathrm{EG}_{4}$ & 52 & 7 & 7.33 & 3 & 6.33 & 3 & 22.66 & 4 & 11.44 & 6 & 66.88 & 13 & 19.00 & 19 & 4.95 & 18 & 1.05 & 6 & 102.0 & 1 & 4.10 & 3 & 6 \\
\hline 5 & $\mathrm{DE}_{1}$ & 50 & 6 & 4.66 & 5 & 4.66 & 5 & 20.66 & 5 & 12.66 & 10 & 86.44 & 3 & 23.88 & 10 & 5.24 & 12 & 1.00 & 9 & 93.22 & 7 & 4.04 & 5 & 5 \\
\hline 6 & $\mathrm{DE}_{2}$ & 47 & 4 & 4.00 & 7 & 3.00 & 10 & 12.66 & 10 & 13.22 & 11 & 96.55 & 1 & 29.60 & 1 & 6.16 & 1 & 1.04 & 7 & 87.44 & 11 & 3.75 & 11 & 4 \\
\hline 7 & $\mathrm{DE}_{3}$ & 45 & 2 & 4.00 & 7 & 2.66 & 11 & 8.66 & 13 & 13.44 & 12 & 65.88 & 15 & 21.11 & 16 & 5.10 & 15 & 0.86 & 15 & 80.88 & 16 & 3.97 & 6 & 15 \\
\hline 8 & $\mathrm{DE}_{4}$ & 50 & 6 & 5.00 & 4 & 4.33 & 6 & 20.66 & 5 & 9.00 & 2 & 74.77 & 10 & 21.75 & 14 & 5.77 & 6 & 1.13 & 4 & 97.77 & 2 & 3.77 & 9 & 2 \\
\hline 9 & $\mathrm{DE}_{5}$ & 45 & 2 & 4.33 & 6 & 2.00 & 12 & 12.66 & 10 & 9.88 & 4 & 75.55 & 8 & 25.17 & 7 & 5.79 & 5 & 1.26 & 1 & 91.11 & 8 & 3.00 & 21 & 7 \\
\hline 10 & $\mathrm{DE}_{6}$ & 46 & 3 & 4.00 & 7 & 3.33 & 9 & 13.33 & 9 & 11.55 & 7 & 86.22 & 4 & 25.35 & 5 & 5.81 & 4 & 1.19 & 2 & 96.77 & 3 & 3.19 & 19 & 3 \\
\hline 11 & $\mathrm{DE}_{7}$ & 45 & 2 & 3.33 & 9 & 3.00 & 10 & 13.33 & 9 & 21.33 & 19 & 81.44 & 5 & 23.04 & 12 & 6.01 & 2 & 1.14 & 3 & 85.00 & 13 & 3.91 & 7 & 9 \\
\hline 12 & $\mathrm{DE}_{8}$ & 47 & 4 & 4.00 & 7 & 3.33 & 9 & 12.00 & 11 & 19.33 & 17 & 69.44 & 12 & 25.54 & 4 & 5.85 & 3 & 0.94 & 13 & 86.77 & 12 & 3.26 & 17 & 12 \\
\hline 13 & $\mathrm{DE}_{9}$ & 48 & 5 & 5.00 & 4 & 3.66 & 8 & 12.66 & 10 & 19.00 & 16 & 54.66 & 19 & 23.42 & 11 & 5.64 & 8 & 0.96 & 11 & 84.22 & 14 & 4.27 & 1 & 11 \\
\hline 14 & $\mathrm{DE}_{10}$ & 59 & 8 & 4.66 & 5 & 4.00 & 7 & 14.66 & 7 & 9.11 & 3 & 60.66 & 17 & 21.13 & 15 & 5.44 & 9 & 0.94 & 13 & 89.33 & 10 & 3.25 & 18 & 13 \\
\hline 15 & $\mathrm{ED}_{1}$ & 48 & 5 & 3.00 & 10 & 2.00 & 12 & 10.00 & 12 & 13.44 & 12 & 65.88 & 15 & 16.00 & 21 & 5.03 & 17 & 0.95 & 12 & 84.00 & 15 & 3.28 & 16 & 20 \\
\hline 16 & $\mathrm{ED}_{2}$ & 50 & 6 & 3.66 & 8 & 1.33 & 13 & 6.00 & 15 & 15.11 & 14 & 56.33 & 18 & 25.24 & 6 & 5.30 & 11 & 0.78 & 17 & 72.55 & 20 & 4.06 & 4 & 16 \\
\hline 17 & $\mathrm{ED}_{3}$ & 48 & 5 & 3.00 & 10 & 2.66 & 11 & 8.00 & 14 & 12.44 & 9 & 62.55 & 16 & 24.35 & 8 & 5.12 & 14 & 0.90 & 14 & 76.33 & 18 & 3.15 & 20 & 18 \\
\hline 18 & $\mathrm{ED}_{4}$ & 50 & 6 & 3.66 & 8 & 3.33 & 9 & 10.00 & 12 & 13.44 & 12 & 44.88 & 21 & 20.48 & 17 & 5.36 & 10 & 0.96 & 11 & 73.44 & 19 & 3.47 & 15 & 19 \\
\hline 19 & $\mathrm{ED}_{5}$ & 50 & 6 & 4.00 & 7 & 4.00 & 7 & 14.00 & 8 & 12.00 & 8 & 46.44 & 20 & 21.97 & 13 & 5.08 & 16 & 0.96 & 11 & 70.66 & 22 & 3.51 & 14 & 16 \\
\hline 20 & E & 42 & 1 & 4.00 & 7 & 3.66 & 8 & 25.33 & 2 & 18.11 & 15 & 66.33 & 14 & 26.40 & 3 & 5.17 & 13 & 0.98 & 10 & 78.88 & 17 & 3.81 & 8 & 10 \\
\hline 21 & D & 48 & 5 & 4.66 & 5 & 3.66 & 8 & 32.00 & 1 & 20.44 & 18 & 75.11 & 9 & 19.80 & 18 & 4.88 & 19 & 1.10 & 5 & 71.33 & 21 & 3.66 & 13 & 14 \\
\hline 22 & G & 52 & 7 & 5.00 & 4 & 4.66 & 5 & 23.33 & 3 & 23.04 & 20 & 77.02 & 7 & 15.74 & 22 & 3.27 & 22 & 0.82 & 16 & 94.76 & 6 & 2.83 & 22 & 17 \\
\hline
\end{tabular}

Note: EG (Brassica napus x Brassica juncea); DE (Brassica napus x Brassica napus); ED (Brassica napus x Brassica napus) 
Table 4. Spearman's rank correlations among yield related characters of 22 genotypes of Brassica spp.

\begin{tabular}{|c|c|c|c|c|c|c|c|c|c|c|}
\hline Characters & $\begin{array}{c}\text { No. of } \\
\text { flower set } \\
\text { per plant } \\
\text { (self) }\end{array}$ & $\begin{array}{l}\text { Total no. } \\
\text { siliqua per } \\
\text { plant } \\
\text { (self) }\end{array}$ & $\begin{array}{c}\text { No. of } \\
\text { seeds per } \\
\text { siliqua } \\
\text { (self) }\end{array}$ & $\begin{array}{c}\text { No. of } \\
\text { flower set } \\
\text { per plant } \\
\text { (open) }\end{array}$ & $\begin{array}{l}\text { Total no. } \\
\text { siliqua per } \\
\text { plant } \\
\text { (open) }\end{array}$ & $\begin{array}{c}\text { No. of } \\
\text { seeds per } \\
\text { siliqua } \\
\text { (open) }\end{array}$ & $\begin{array}{c}\text { Siliqua } \\
\text { length } \\
(\mathrm{cm})\end{array}$ & $\begin{array}{l}\text { Beak } \\
\text { length } \\
(\mathrm{cm})\end{array}$ & $\begin{array}{l}\text { Plant } \\
\text { height } \\
(\mathrm{cm})\end{array}$ & $\begin{array}{c}1000 \\
\text { seed } \\
\text { weight } \\
(\mathrm{gm})\end{array}$ \\
\hline $50 \%$ flowering (days) & 0.281 & $0.441^{*}$ & 0.252 & -0.164 & -0.309 & $-0.546^{* *}$ & -0.302 & -0.317 & 0.103 & -0.012 \\
\hline $\begin{array}{l}\text { No. of flower set per plant } \\
\text { (self) }\end{array}$ & & $0.817 * *$ & $0.607 * *$ & -0.312 & 0.357 & -0.188 & -0.326 & 0.260 & $0.675^{* *}$ & 0.291 \\
\hline $\begin{array}{l}\text { Total no. siliqua per plant } \\
\text { (self) }\end{array}$ & & & $0.768^{* *}$ & -0.372 & 0.300 & -0.208 & -0.412 & 0.235 & $0.556^{* *}$ & 0.263 \\
\hline $\begin{array}{l}\text { No. of seeds per siliqua } \\
\text { (self) }\end{array}$ & & & & $-0.563 * *$ & 0.379 & -0.220 & -0.328 & 0.409 & 0.394 & 0.138 \\
\hline $\begin{array}{l}\text { No. of flower set per plant } \\
\text { (open) }\end{array}$ & & & & & -0.106 & -0.074 & 0.103 & -0.338 & -0.287 & 0.313 \\
\hline $\begin{array}{l}\text { Total no. siliqua per plant } \\
\text { (open) }\end{array}$ & & & & & & 0.261 & 0.101 & $0.577^{* *}$ & $0.625^{* *}$ & -0.005 \\
\hline $\begin{array}{l}\text { No. of seeds per siliqua } \\
\text { (open) }\end{array}$ & & & & & & & $0.536^{*}$ & 0.120 & -0.010 & -0.031 \\
\hline Siliqua length $(\mathrm{cm})$ & & & & & & & & 0.310 & 0.045 & -0.097 \\
\hline $\begin{array}{l}\text { Beak length }(\mathrm{cm}) \\
\text { Plant height }(\mathrm{cm})\end{array}$ & & & & & & & & & $0.477 *$ & $\begin{array}{l}0.118 \\
0.056\end{array}$ \\
\hline
\end{tabular}

$*$ and $* *$ indicate significant at $5 \%$ and $1 \%$ level of probability, respectivel 
Highly significant negative correlation was found between number of flower set per plant and siliqua length $\left(r=-0.473^{*}\right)$ (Table 2). Total number of siliqua per plant (self) had highly significant positive correlation with plant height $\left(\mathrm{r}=0.553^{* *}\right)$ and significant negative correlation with number of flower set per plant $(r=-0.454 *)$. Significant positive correlation was found between total number of siliqua set per plant (self) and number of seeds per siliqua (self) $\left(r=0.486^{*}\right)$ and significant negative correlation was found between total number of siliqua set per plant (self) and siliqua length $\left(r=-0.489^{*}\right)$ (Table 2). Non-significant positive correlation of number of seeds per siliqua (self) was found with beak length $(r=0.298)$, plant height $(r=0.261)$ and 1000-seed weight $(r=$ 0.110 ). Non-significant negative correlation of number of seeds per siliqua (self) was found with siliqua length $(\mathrm{r}=-0.363)$ (Table 2$)$. Further, highly positive correlation was observed between total number of siliqua per plant (open) and plant height $\left(\mathrm{r}=0.608^{* *}\right)$. Total number of siliqua per plant (open) had significant positive correlation with beak length $\left(\mathrm{r}=0.488^{*}\right)$. Kashyap and Mishra (2004), Mahak et al. (2003), Pant et al. (2002) reported positive and significant correlation between number of siliqua per plant and seed yield per plant. Number of seeds per siliqua (open) had highly significant positive correlation with siliqua length $\left(\mathrm{r}=0.527^{*}\right)$ (Table 2$)$. The present findings showed conformity with those of Pant et al. (2002). Lebowitz (1989) found siliqua length was positively correlated with number of seeds per siliqua $(r=0.602)$. Siliqua length had insignificant positive correlation with beak length $(\mathrm{r}=0.404)$ and 1000 -seed weight $(\mathrm{r}=$ $0.142)$ while insignificant negative correlation with plant height $(\mathrm{r}=-0.098)$. On the other hand, plant height positively correlated with beak length $(r=0.422)$ and 1000 -seed weight $(r=0.010)$ while beak length had insignificant negative correlation with 1000seed weight $(\mathrm{r}=-0.004)$ (Table 2). The genotypes ranked on the basis of individual score secured by each of the eleven characters. The total relative ranking ranged from 1 to 20 and the first rank was estimated for the genotype $\mathrm{EG}_{2}$ followed by $\mathrm{DE}_{2}, \mathrm{DE}_{4}$ and $\mathrm{DE}_{6}$ genotypes.

The last rank was estimated for the genotype $\mathrm{ED}_{1}$ (Table 3). In order to compare the method of morphological characterization for distinguishing genotypes, this test was carried out (Table 4). Grouping of genotypes obtained through these rank correlation values were effective and repeatable. In this present study, the Spearman's rank correlation test presented 12 relationships that showed 10 positive significant and 2 negative significant correlations. In normal Coefficients of Correlation, 10 significant associations were found but 12 associations in Spearman's rank correlation. In this Spearman's rank correlation test, $50 \%$ flowering days had significant positive correlation with number of siliqua per plant (self) $\left(\mathrm{r}_{\mathrm{s}}=0.441^{*}\right)$ and also had significant negative correlation with number of seeds per siliqua (open) $\left(r_{s}=-0.546^{* *}\right)$ (Table 4). But in the normal correlation this trait did not show any significant correlation. In these techniques, number of flower set per plant (self) had highly significant positive correlation with total number of siliqua per plant $\left(\mathrm{r}_{\mathrm{s}}=0.817^{* *}\right)$, number of seeds per siliqua (self) $\left(\mathrm{r}_{\mathrm{s}}=0.607 * *\right)$ and plant height $\left(\mathrm{r}_{\mathrm{s}}=0.675^{* *}\right)$. Highly significant positive correlation found in total number of siliqua per plant (self) with number of seeds per siliqua (self) $\left(\mathrm{r}_{\mathrm{s}}=0.768^{* *}\right)$ and plant height $\left(\mathrm{r}_{\mathrm{s}}=0.556^{* *}\right)$ and significant negative correlation with number of flower set per plant (open) $\left(r_{s}=-0.563 * *\right)$ (Table 4). Total number of siliqua per plant (open) had highly significant positive correlation with plant height $\left(\mathrm{r}_{\mathrm{s}}=0.625^{* *}\right)$ and beak length $\left(\mathrm{r}_{\mathrm{s}}=0.577^{* *}\right)$, which showed the similar results in normal Coefficients of Correlation. Number of seeds per siliqua (open) and beak length both had showed the significant positive correlation in siliqua length $\left(\mathrm{r}_{\mathrm{s}}=0.536^{*}\right)$ and 
plant height $\left(r_{s}=0.477^{*}\right)$, respectively. Among the cross products of $\mathrm{C}_{7}$ and $\mathrm{F}_{5}$ generations with their parents, inter-species $\mathrm{EG}_{2}$ and $\mathrm{EG}_{1}$ showed better agronomic performance. Phenotypic characters of the genotypes were closely associated with each other both normal Correlation Coefficients and Spearman's Rank Correlation Coefficients. The total relative ranking ranged from 1 to 20 and the first rank was estimated for the genotype $\mathrm{EG}_{2}$ genotypes. The last rank was estimated for the genotype $\mathrm{ED}_{1}$. Out of 55 associations, which found in the correlation study, 10 associations were found highly significant at the morphological level. This genetic study indicated that there is almost clearly defined variation in the parents and their cross, and reciprocal cross products. And definitely this research might have a remarkable impact to increase knowledge of plant breeders for future sustainable Brassica spp. breeding program.

\section{REFERENCES}

AIS. 2013. Agricultural Information Service. Krishi Diary (In Bangla).Agril.Inform. Ser.Khamarbari, Farmgate, Dhaka, Bangladesh. P. 16.

BBS. 2011. Year Book of Agricultural Statistics of Bangladesh. Bangladesh Bureau of Statistics, Ministry of Planning. Govt. People's Republic of Bangladesh. P. 38332.

Begum, F., F. Hossain and M. R. I. Mondol. 2012. Influence of sulphur on morphophysiological and yield parameters of rapeseed. Bangladesh J. Agril. Res. 37(4): 645-652.

Kashyap, S. C. and M. N. Mishra. 2004. Correlation and Path coefficient analysis studies in toria ( B. campestris Var. toria). Annu. Agril. Bio. Res. 9(2): 123-126.

Labowitz, R. J. 1989. Image analysis measurements of repeatability estimates of siliqua morphological traits in B. campestris L. Eupytica. 43(1-2): 113-116.

Mahak, S., G. B. Lallu, L. P. Swarnkar and R. K. Dixit. 2003. Heterobeltiosis in Indian mustard (B. juncea L. Czern and Coss). Crop Res. Hisar. 26(2): 318-325.

Naz, S., L. Rahman, M. A. Quddus and U. K. Nath. 2003. Correlation and path analysis in parent, intervariety and interspecies hybrids of $B$. juncea and B. campestris. Bangladesh J. Agril. Sci. 30(1): 169-172.

Pant, S. C., P. Singh, R. Kumar, S. Mishra, S. P. Singh and S. Mishra. 2002. Correlation and path analysis in Indian mustard. Plant-Archives. 2(2):207-211. 